\title{
Response to neoadjuvant treatment among rectal cancer patients in a population-based cohort
}

\author{
Elizabeth Alwers ${ }^{1} \cdot$ Lina Jansen ${ }^{1}$ - Jakob Kather ${ }^{2}$ • Efrat Amitay ${ }^{1} \cdot$ Hendrik Bläker $^{3}$ - Matthias Kloor ${ }^{4}$. \\ Katrin E. Tagscherer ${ }^{5,6}$. Wilfried Roth ${ }^{5,6} \cdot$ Esther Herpel $^{5,7}$ • Jenny Chang-Claude ${ }^{8,9} \cdot$ Hermann Brenner $^{1,10,11}$. \\ Michael Hoffmeister ${ }^{1}$
}

Accepted: 8 September 2020 / Published online: 19 September 2020

(C) The Author(s) 2020

\begin{abstract}
Background In rectal cancer, prediction of tumor response and pathological complete response (pCR) to neoadjuvant treatment could contribute to refine selection of patients who might benefit from a delayed- or no-surgery approach. The aim of this study was to explore the association of clinical and molecular characteristics of rectal cancer with response to neoadjuvant treatment and to compare patient survival according to level of response.

Methods Resected rectal cancer patients were selected from a population-based cohort study. Molecular tumor markers were determined from the surgical specimen. Tumor response and pCR were defined as downstaging in T or N stage and absence of tumor cells upon pathological examination, respectively. The associations of patient and tumor characteristics with tumor response and pCR were explored, and patient survival was determined by degree of response to neoadjuvant treatment.

Results Among 1536 patients with rectal cancer, $602(39 \%)$ received neoadjuvant treatment. Fifty-five (9\%) patients presented pCR, and $239(49 \%)$ and $250(53 \%)$ patients showed downstaging of the $\mathrm{T}$ and $\mathrm{N}$ stages, respectively. No statistically significant associations were observed between patient or tumor characteristics and tumor response or pCR. Patients who presented any type of response to neoadjuvant treatment had significantly better cancer-specific and overall survival compared with non-responders. Conclusion In this study, patient characteristics were not associated with response to neoadjuvant treatment, and molecular characteristics determined after surgical resection of the tumor were not predictive of $\mathrm{pCR}$ or tumor downstaging. Future studies should include molecular biomarkers from biopsy samples before neoadjuvant treatment.
\end{abstract}

Keywords Rectal cancer $\cdot$ Neoadjuvant treatment $\cdot$ Response to treatment $\cdot$ Pathological complete response

\section{Introduction}

Neoadjuvant treatment is recommended for patients with locally advanced rectal cancer, usually including chemo-/radiotherapy (nCRT) followed by surgical resection [1]. The surgical approach, including abdominoperineal resection and total mesorectal excision, yields good local control and prognosis;

Electronic supplementary material The online version of this article (https://doi.org/10.1007/s00384-020-03744-2) contains supplementary material, which is available to authorized users.

Elizabeth Alwers

Elizabeth.alwers@dkfz.de

Extended author information available on the last page of the article however, it is also associated with significant peri-operative morbidity, including bowel, sexual and urinary dysfunctions [2], and a permanent colostomy in some cases, which severely affects the patient's quality of life [3,4].

To spare rectal cancer patients from the morbidity caused by surgical resection, the watch-and-wait approach has been suggested [5]. This approach proposes that surgery be delayed in patients who achieve a clinical complete response (cCR: no evidence of tumor in regular clinical and radiological examinations) after receiving neoadjuvant treatment $[5,6]$. Case series and cohort studies have shown benefits of a watchand-wait approach after cCR, resulting in organ preservation and equivalent oncological outcomes as compared with those of patients who achieved pathological complete response (pCR: no evidence of residual tumor cells upon pathological examination) after radical surgery [7-9]. Optimal selection of 
patients who are eligible for a watch-and-wait approach continues to be challenging, given that no definitive predictors of pCR have been identified [10].

Among the established molecular markers of colorectal cancer, KRAS and TP53 mutations have been associated with lack of response to neoadjuvant treatment in observational studies with small sample sizes [11, 12], while evidence for other molecular markers is still scarce [13]. Some studies have indicated that post-surgical mutations of CRC might be representative of those present in the tumor prior to administration of nCRT [14]. The aim of this study was to compare patient and tumor characteristics of rectal cancer patients according to administration of neoadjuvant treatment. Also, we aimed to describe clinical and molecular predictors of tumor response and pCR among rectal cancer patients who received neoadjuvant treatment and describe survival among responders and non-responders.

\section{Methods}

\section{Study design and patient selection}

The DACHS (Darmkrebs: Chancen der Verhütung durch Screening) study is a population-based case-control study conducted since 2003 in more than twenty hospitals in the south-west of Germany that was initiated to investigate the potential of screening endoscopy in the reduction of colorectal cancer risk. In addition, patients with colorectal cancer are followed up as a cohort at 3,5, and 10 years after diagnosis [15-17]. In this analysis, patients diagnosed with rectal cancer (ICD-10 code C20) between 2003 and 2014 were included. Baseline socio-demographic and lifestyle characteristics, as well as medical and family history, were obtained by trained interviewers using a standardized questionnaire at the time of diagnosis. Clinical and pathological characteristics were determined from medical records, pathology reports, and hospital discharge letters after surgical resection of the tumor. Information on type of therapy, comorbidities, and recurrence of disease was determined from medical reports obtained from the treating physicians. Long-term follow-up was performed at 3,5 , and 10 years after diagnosis including information on recurrence of disease. Vital status and date and cause of death were determined from population registries and death certificates issued by the health authorities.

Only patients with stage II or III rectal cancer at diagnosis who underwent surgical resection of the tumor were included in this analysis. The type of surgical resection and neoadjuvant treatment schemes were provided according to general clinical guidelines and the treating physician's decision. Molecular tumor characterization was available for a subset of these patients (see patient selection in supplementary figure 1). Molecular markers, including KRAS mutations and $\mathrm{CpG}$ island methylator phenotype (CIMP) status, were determined in tumor tissue specimens after surgical resection and performed on formalin-fixed, paraffin-embedded (FFPE) samples, as previously described [15].

\section{Tumor response and pathological complete response}

Tumor characteristics including $\mathrm{T}$ and $\mathrm{N}$ stages, grade, and histology were derived from post-surgical pathology reports. Outcomes of interest included the difference between clinical (cT, $\mathrm{cN}$ ) and pathological (ypT, ypN) $\mathrm{T}$ and $\mathrm{N}$ stages after neoadjuvant treatment. Tumor response was defined as downstaging of $\mathrm{T}, \mathrm{N}$, or both stages among patients with available information (i.e., excluding TX, NX). pCR was defined as ypT0-N0 stage determined from pathology reports after neoadjuvant treatment.

\section{Patient survival}

Secondary outcomes included cancer-specific survival (CSS), defined as time from diagnosis until death from rectal cancer; relapse-free survival (RFS), defined as time from diagnosis until reappearance of disease, metastases, cancer death, or death from other causes; and overall survival (OS), defined as time from diagnosis until death from any cause. The association between $\mathrm{pCR}$ and tumor response (downstage in $\mathrm{T}$ or $\mathrm{N}$ ) with survival outcomes was studied among patients who received neoadjuvant treatment adjusting for relevant covariates such as age, clinical stage at diagnosis, adjuvant chemotherapy, and comorbidities (Charlson comorbidity score $[18,19])$. In a complementary analysis, CSS, RFS, and OS were determined independently for patients who received neoadjuvant treatment and presented response or not, and compared with patients who did not receive neoadjuvant treatment.

\section{Statistical analysis}

Socio-demographic characteristics were described for the entire patient population and stratified by whether the patient received neoadjuvant treatment or not. Chi-square or Fisher's exact tests were used to explore the associations between patient and tumor characteristics, and the specified outcomes of tumor response and pCR. Tumor response, expressed as the change in $\mathrm{T}$ and $\mathrm{N}$ stages, was also visually explored by means of alluvial diagrams. Cox proportional hazard models were used to estimate HR and $95 \%$ CI for the secondary survival outcomes. All statistical analyses were performed using R version 3.5.1, packages dplyr, survival, and ggalluvial [20]. 


\section{Results}

\section{Patient and tumor characteristics}

Overall, 1536 patients with resected rectal cancer were included in this analysis, of whom 602 (39\%) received neoadjuvant treatment. Table 1 presents patient and tumor characteristics, overall and by administration of neoadjuvant treatment or not. Overall, a larger proportion of patients $(66 \%)$ was male, and median age at diagnosis was 68 years for patients who did not receive neoadjuvant treatment and 65 years for those who did. Among patients who received neoadjuvant treatment, 90\% presented with clinical T3/T4 tumors and $71 \%$ with any type of lymph node involvement (N+/N1-3). The use of neoadjuvant treatment increased by year of diagnosis: $32 \%$ of patients diagnosed between 2003 and 2006 received neoadjuvant treatment, while this proportion increased to $45 \%$ for patients diagnosed between 2011 and 2014.

\section{Molecular tumor markers}

Information on tumor molecular characteristics was available for $682(44 \%)$ patients, of whom $212(31 \%)$ received neoadjuvant treatment (see Table 1). KRAS mutations were found in $144(34 \%)$ patients who did not receive neoadjuvant

Table 1 Rectal cancer patient and tumor characteristics by neoadjuvant treatment $-n(\%)$

\begin{tabular}{|c|c|c|c|c|}
\hline Variable & Category & $\begin{array}{l}\text { Overall } \\
(n=1536)\end{array}$ & $\begin{array}{l}\text { No neoadjuvant } \\
(n=934)\end{array}$ & $\begin{array}{l}\text { Neoadjuvant } \\
(n=602)\end{array}$ \\
\hline \multirow[t]{3}{*}{ Age group } & $\leq 64$ & $656(42.7)$ & $366(39.2)$ & $290(48.2)$ \\
\hline & $65-74$ & $520(33.9)$ & $315(33.7)$ & $205(34.1)$ \\
\hline & $\geq 75$ & $360(23.4)$ & $253(27.1)$ & $107(17.8)$ \\
\hline \multirow[t]{2}{*}{ Gender } & Female & $521(33.9)$ & $344(36.8)$ & $177(29.4)$ \\
\hline & Male & $1015(66.1)$ & $590(63.2)$ & $425(70.6)$ \\
\hline \multirow[t]{3}{*}{ Comorbidity index } & 0 & $899(58.5)$ & $518(55.5)$ & $381(63.3)$ \\
\hline & 1 & $287(18.7)$ & $179(19.2)$ & $108(17.9)$ \\
\hline & $2-3$ & $350(22.8)$ & $237(25.4)$ & $113(18.8)$ \\
\hline \multirow[t]{3}{*}{ Year of diagnosis } & 2003-2006 & $514(31.6)$ & $351(35.3)$ & $163(25.8)$ \\
\hline & 2007-2010 & $491(30.2)$ & $290(29.2)$ & $201(31.8)$ \\
\hline & 2011-2014 & $531(32.7)$ & $293(29.5)$ & $238(37.6)$ \\
\hline \multirow[t]{5}{*}{ Clinical T stage } & $\mathrm{T} 1$ & $54(6.2)$ & $54(15.0)$ & 0 \\
\hline & $\mathrm{T} 2$ & $151(17.4)$ & $113(31.4)$ & $38(7.5)$ \\
\hline & $\mathrm{T} 3$ & $524(60.3)$ & $132(36.7)$ & $392(77.0)$ \\
\hline & $\mathrm{T} 4$ & $90(10.4)$ & $24(6.7)$ & $66(13.0)$ \\
\hline & $\mathrm{TX}$ & $50(5.8)$ & $37(10.3)$ & $13(2.6)$ \\
\hline \multirow[t]{6}{*}{ Clinical N stage } & No & $301(35.6)$ & $175(50.1)$ & $126(25.4)$ \\
\hline & $\mathrm{N}+$ & $231(27.3)$ & $37(10.6)$ & $194(39.1)$ \\
\hline & N1 & $156(18.5)$ & $49(14.0)$ & $107(21.6)$ \\
\hline & N2 & $65(7.7)$ & $17(4.9)$ & $48(9.7)$ \\
\hline & N3 & $3(0.4)$ & $0(0.0)$ & $3(0.6)$ \\
\hline & NX & $89(10.5)$ & $71(20.3)$ & $18(3.6)$ \\
\hline \multirow[t]{5}{*}{$\mathrm{T}$ stage after treatment* } & T0 & - & - & $61(10.2)$ \\
\hline & Tis-T1 & - & $208(22.3)$ & $33(5.5)$ \\
\hline & $\mathrm{T} 2$ & - & $245(26.3)$ & $151(25.3)$ \\
\hline & $\mathrm{T} 3$ & - & $424(45.5)$ & $327(54.8)$ \\
\hline & $\mathrm{T} 4$ & - & $55(5.9)$ & $25(4.2)$ \\
\hline \multirow[t]{3}{*}{$\mathrm{N}$ stage after treatment* } & No & - & $571(63.9)$ & $404(67.7)$ \\
\hline & N1 & - & $190(21.3)$ & $131(21.9)$ \\
\hline & N2 & - & $132(14.8)$ & $62(10.4)$ \\
\hline \multicolumn{2}{|c|}{ Patients with available tumor marker characterization } & Overall $(n=682)$ & No neoadjuvant $(n=470)$ & Neoadjuvant $(n=212)$ \\
\hline \multirow[t]{2}{*}{ KRAS mutation } & Non-mut & $432(68.9)$ & $286(66.5)$ & $146(74.1)$ \\
\hline & Mutated & $195(31.1)$ & $144(33.5)$ & $51(25.9)$ \\
\hline \multirow[t]{2}{*}{ CIMP status } & Low/neg & $613(91.2)$ & $419(90.3)$ & $194(93.3)$ \\
\hline & High & $59(8.8)$ & $45(9.7)$ & $14(6.7)$ \\
\hline
\end{tabular}

Totals may not add up because of missing values. Clinical T stage information unavailable for 694 patients (14\% received neoadjuvant). Clinical $\mathrm{N}$ stage information unavailable for 718 patients (15\% received neoadjuvant). Missing $\mathrm{T}$ and $\mathrm{N}$ information for 2 and 41 patients who did not receive neoadjuvant treatment, respectively. Missing KRAS status $n=59$ (27\% received neoadjuvant), and missing CIMP $n=10$ ( 4 received neoadjuvant). Missing $\mathrm{T}$ and $\mathrm{N}$ stage information for 5 patients who received neoadjuvant treatment

* $\mathrm{T}$ and $\mathrm{N}$ stages were determined from pathology reports after surgery for patients who did not receive neoadjuvant treatment $(\mathrm{pT}$ and $\mathrm{pN})$ and patients who received neoadjuvant treatment (ypT and ypN) 
treatment and in $51(26 \%)$ patients who received neoadjuvant treatment $(p=0.069)$.

\section{Response to neoadjuvant treatment}

Among 602 patients who received neoadjuvant treatment, 61 (10\%) had no evidence of residual tumor after surgical resection (ypT0), 33 (5\%) had ypTis-T1 tumors, 151 (25\%) had ypT2, and 327 (55\%) showed ypT3 tumors. After neoadjuvant treatment, $404(68 \%)$ patients had ypN0 stage, while 131 $(22 \%)$ and $62(10 \%)$ showed ypN1 and ypN2 diseases, respectively (see Table 1).

Table 2 presents outcomes of response to treatment among patients who received neoadjuvant therapy. pCR, defined as ypT0N0 stage after neoadjuvant treatment, was observed in $55(9 \%)$ patients. Among patients with available information, $233(48 \%)$ had no change in the $\mathrm{T}$ stage after neoadjuvant treatment, while 239 (49\%) presented downstaging of the tumor, and $19(4 \%)$ a higher T stage. Similarly, 250 (53\%) patients presented a reduction in $\mathrm{N}$ stage, 186 (39\%) no improvement, and $37(8 \%)$ a higher $\mathrm{N}$ stage than was clinically assessed. The transition of patients from clinical stages (cT, $\mathrm{cN})$ to pathological stages (ypT, ypN) is additionally presented in Fig. 1.

Table 3 presents patient and tumor marker characteristics by outcomes of pCR and tumor response among patients who received neoadjuvant treatment. No statistically significant associations were observed between the investigated characteristics

Table 2 Tumor response among rectal cancer patients who received neoadjuvant treatment $(n=602)$

\begin{tabular}{lll}
\hline Outcome & Category & $n(\%)$ \\
\hline pCR (ypT0, ypN0) & No & $539(90.7)$ \\
Change in T stage (ypT-cT) & Yes & $55(9.3)$ \\
& -3 & $5(1.0)$ \\
& -2 & $45(9.2)$ \\
Any change in T stage & -1 & $39(7.9)$ \\
& 0 & $150(30.6)$ \\
Any change in N stage & 1 & $233(47.5)$ \\
& Decreased & $239(48.9)$ \\
& Same & $233(47.5)$ \\
& Increased & $19(3.9)$ \\
& Decreased & $250(52.9)$ \\
& Same & $186(39.3)$ \\
& Increased & $37(7.8)$ \\
\hline
\end{tabular}

$p C R$, pathological complete response (ypT0, ypN0)

Change in $\mathrm{T}$ stage calculated as the difference between ypT stage (determined from surgical specimen) and cT stage, for patients with available information $(n=491)$ and tumor response. Patient clinical characteristics did not show any relevant associations to predict pCR or tumor downstaging in univariate analyses. Patients who presented downstaging in the number of involved lymph nodes or showed any response to neoadjuvant treatment had a somewhat lower, although not significant, proportion of KRAS mutations compared with those who showed no improvement (17\% vs $24 \%, p=0.441$ and $22 \%$ vs $30 \%, p=0.379$, respectively).

\section{Patient survival}

Overall, 210 (35\%) patients died among those who received neoadjuvant treatment and $342(37 \%)$ among those who did not, $146(70 \%)$ and $179(52 \%)$ of whom died of rectal cancer, respectively. Among 55 patients with pCR, 4 (7\%) developed recurrence of disease and died of rectal cancer, and 3 additional patients died from other causes. Among patients with downstaging in $\mathrm{T}$ stage and among those with downstaging in N stage, 43 (18\%) and $52(21 \%)$ presented recurrence of disease, of whom 36 and 46 died of rectal cancer, respectively. In general, presenting any type of response to neoadjuvant treatment was associated with significantly better survival (see supplementary table 1). In a complementary analysis, patients who responded to neoadjuvant treatment (downstaging in $\mathrm{T}, \mathrm{N}$, or pCR) had significantly better survival compared with patients who did not receive neoadjuvant treatment. In contrast, among patients who received neoadjuvant treatment and did not have pCR or did not present downstaging in $\mathrm{T}$ or $\mathrm{N}$, no significant associations were observed with survival. Among patients who presented no response (neither $\mathrm{T}$ nor N), survival was significantly worse compared with patients who did not receive neoadjuvant treatment (see supplementary table 2).

\section{Discussion}

In this population-based cohort study, outcomes of $\mathrm{pCR}$ and tumor response to neoadjuvant treatment were analyzed among resected rectal cancer patients. Among patients who received neoadjuvant treatment, almost half (49\%) presented downstaging of the $\mathrm{T}$ stage of the tumor. This finding reflects the known benefits of neoadjuvant treatment, with reported downstaging rates between 50 and 60\% [21, 22]. Among patients who presented with clinical lymph node involvement, more than half $(52 \%)$ of patients were free of affected lymph nodes after neoadjuvant treatment and resection (ypN0). In this study, $9 \%$ of patients presented pCR; this finding compares with other studies that have reported rates around $10 \%$ (range 8-24\%) [7, 21, 23-26].

Several molecular biomarkers to predict response to neoadjuvant treatment have been studied; however, none so far 
a

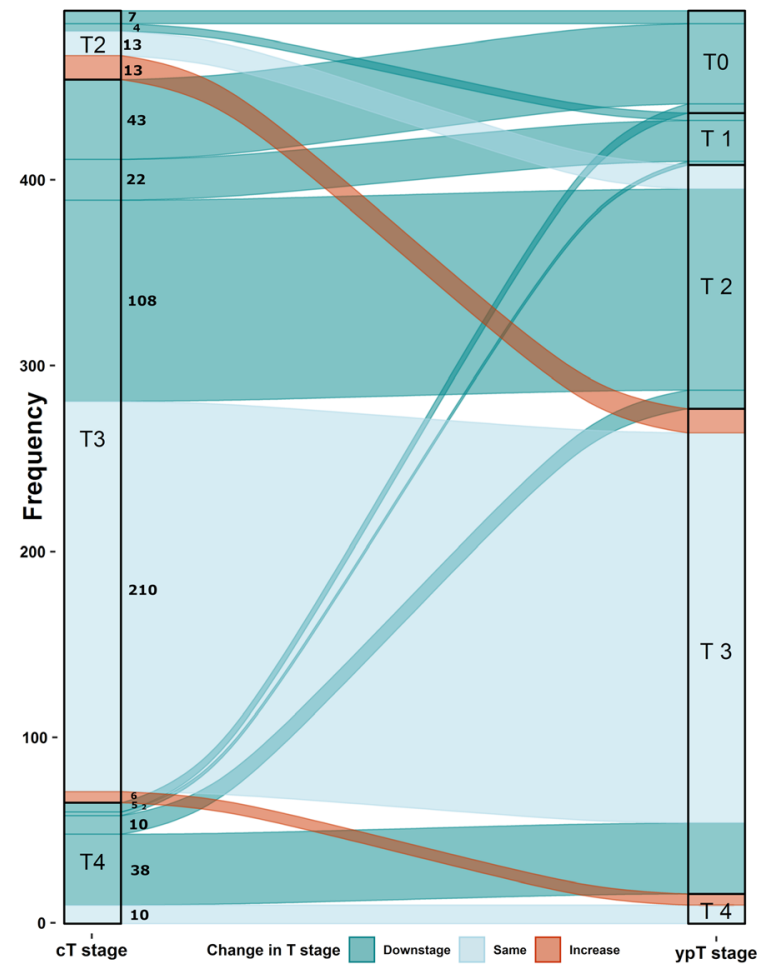

Fig. 1 a Change in $\mathrm{T}$ stage among patients who received neoadjuvant treatment. The left column indicates the proportion of patients in each clinical $\mathrm{T}$ stage (cT stage) category; the right column indicates the proportion of patients in each $\mathrm{T}$ stage after treatment (ypT stage). $\mathbf{b}$ Change in $\mathrm{N}$ stage among patients who received neoadjuvant treatment. The left column indicates the proportion of patients in each clinical $\mathrm{N}$

has predicted $\mathrm{pCR}$ or tumor response to a degree where they could be implemented in clinical practice $[27,28]$. In this study, no associations between major molecular characteristics of the tumor and pCR or tumor downstaging were observed. However, among patients who presented response to nCRT and among those who presented downstaging in the number of lymph nodes, the proportion of KRAS mutations was somewhat lower. The number of patients with CIMP-hi tumors was extremely low, and thus no further analyses could be performed on this marker. Previous studies have reported conflicting results about the role of KRAS as a predictive biomarker of response to nCRT. A retrospective study of biopsies from stage II/III rectal cancer patients reported lower rates of pCR for tumors with KRAS mutations and higher rates of lymph node metastasis for tumors with both KRAS and TP53 mutations [11]. Other studies, however, have reported no such associations [29, 30]. Previous studies have suggested that there may be a difference in the response levels of KRAS mutations occurring in codons 12 and 13 (as investigated in this study) and other less frequent mutations occurring in other codons (e.g., 61 and 146) [29, 31]. Resistance to nCRT may also be influenced by intra-tumoral heterogeneity, b

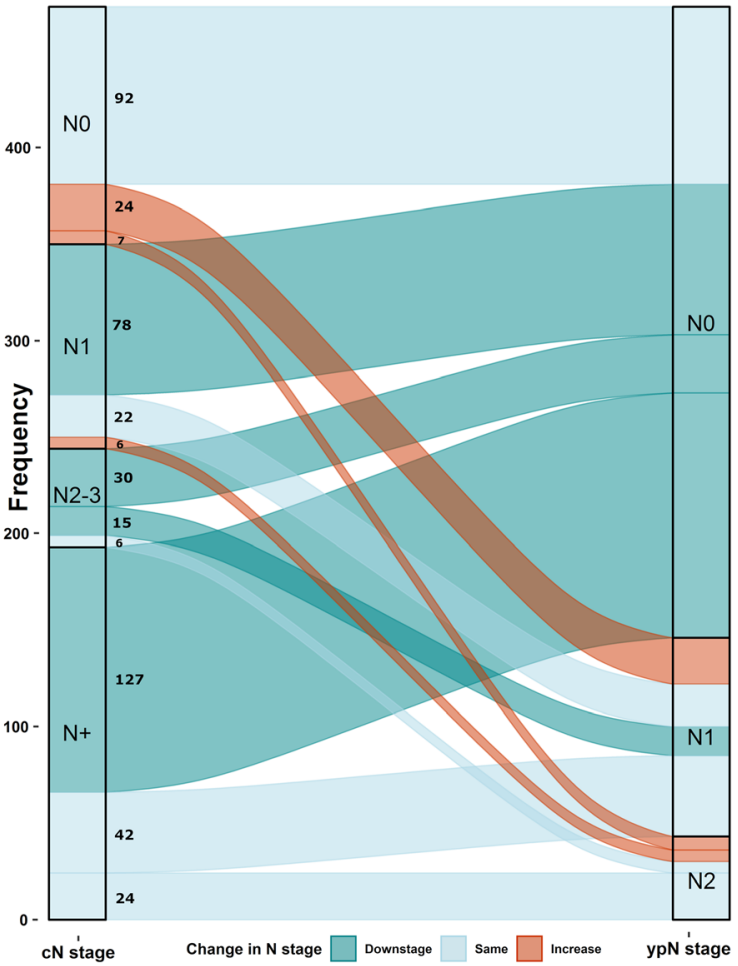

stage ( $\mathrm{cN}$ stage) category; the right column indicates the proportion of patients in each stage after treatment (ypN stage). The flow in between columns indicates the number of patients that transition between categories: dark green indicates downstaging, light blue indicates no change, and red indicates increase in stage

given that different tumor sub-clones might present a variety of mutational and copy number alterations that may differentially respond to treatment [32].

Because all molecular determinations were performed on tumor specimens resected after nCRT, the association between KRAS status and pCR (ypT0-ypN0) could not be analyzed in this study. This constitutes a limitation of this analysis; however, no meaningful differences were observed in the proportion of KRAS mutations identified among patients who received nCRT and those who did not, which constitutes an interesting finding that could be of value for future research on this topic. Previous models have suggested that major genetic mutations that occur early during the tumor development are likely to persist as the tumor grows [14], indicating that the molecular alterations identified after surgical excision may still be representative of those found in the tumor before administration of nCRT. This has also been suggested by a retrospective study among 47 stage II/III rectal adenocarcinoma patients, in which genetic mutations were analyzed on tumor samples before and after nCRT and no significant differences were observed [12]. However, larger studies are needed to confirm this, given that among tumors that respond to 


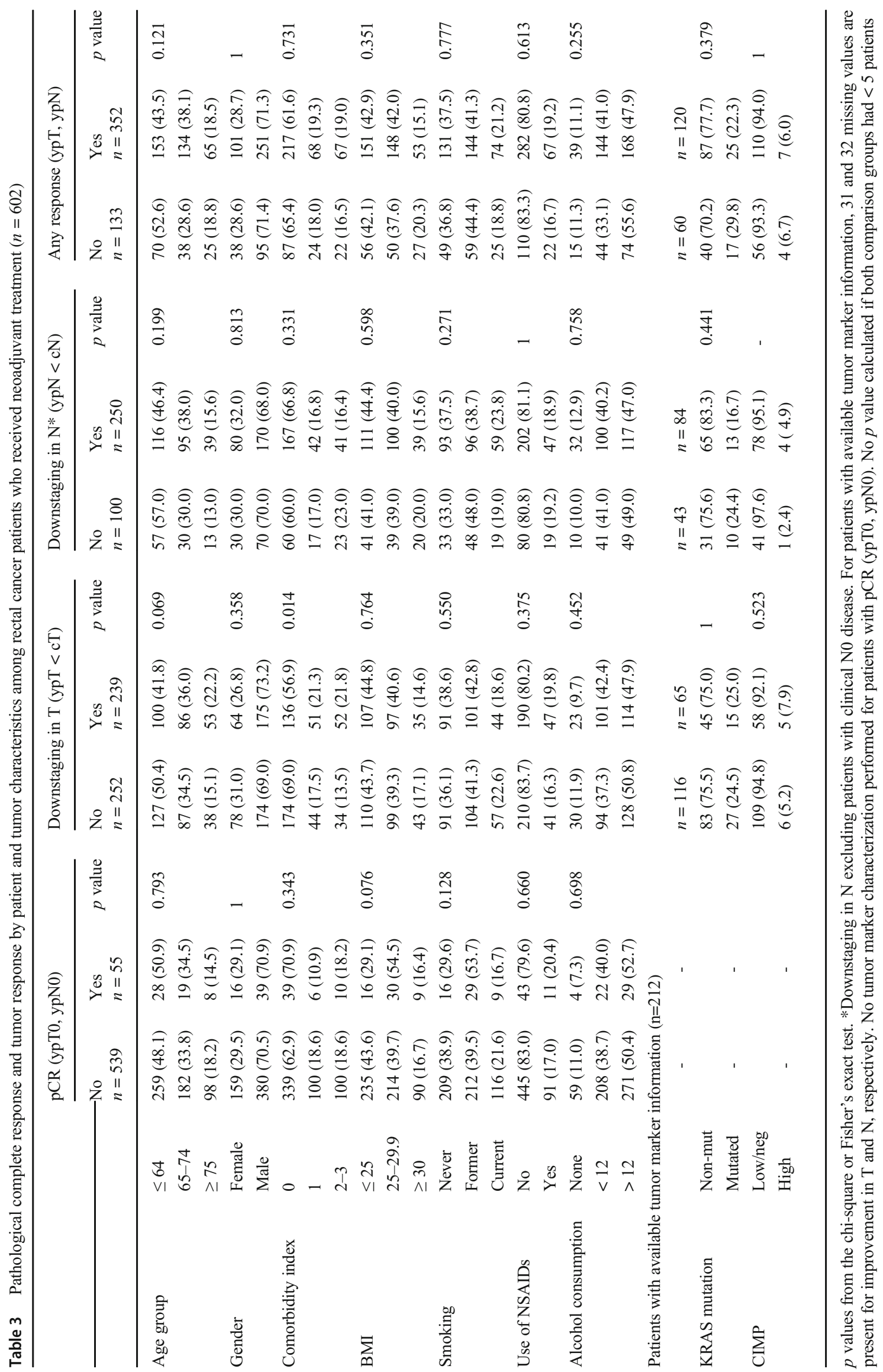


nCRT, the original genetic mutations may no longer be detected after surgical excision. Therefore, future studies should investigate markers identified from biopsy samples to predict response to $\mathrm{nCRT}$.

Inaccuracies in clinical staging might have resulted in larger observed downstaging in some cases, which might be regarded as a limitation of the study. To reduce the impact of such clinical staging inaccuracy, all patients with ambiguous $\mathrm{cT}$ or $\mathrm{cN}$ status were excluded from the analyses. Similarly, patients with TX or NX status were excluded when calculating changes in $\mathrm{T}$ or $\mathrm{N}$ stage, respectively.

Both tumor response and pCR have been identified as important predictors of survival outcomes in rectal cancer [33]. Results from a clinical trial among 400 resected rectal cancer patients who received neoadjuvant chemoradiation showed that tumor regression and $\mathrm{ypN}$ status were independent predictors of disease-free survival and relapse, and local recurrence, respectively $[34,35]$. Other retrospective studies and meta-analyses have reported similar results $[23,24]$. In this study, the survival benefit of patients who responded to nCRT was confirmed. Moreover, in exploratory analyses, nonresponders had a tendency towards worse survival when compared with patients who did not receive nCRT, potentially reflecting tumors of a more aggressive nature.

Given the good prognosis of patients who respond to neoadjuvant treatment and the morbidity associated with rectal cancer surgery, the watch-and-wait approach has been proposed for patients who present cCR after nCRT $[5,36,37]$. Some studies have reported that $\mathrm{cCR}$ is not a perfect predictor of pCR, and therefore, patient selection should not be based solely on clinical absence of the tumor $[38,39]$. Other studies have observed a trend towards better pCR and higher tumor downstaging when the time interval between nCRT and surgery is increased to between 8 and 10 weeks [26]. This indicates that waiting for a longer period after nCRT to evaluate response to treatment might be beneficial in sparing surgery for some patients. To date, no evidence from randomized clinical trials is available to recommend the watch-and-wait approach; however, several ongoing clinical trials will contribute to clarify this question (e.g., NCT02704520, NCT02008656, and NCT02945566). Because results on long-term follow-up and survival outcomes for these patients will likely take long time to be published, evidence from retrospective studies is still considered relevant to suggest potential molecular biomarkers that could be validated in larger studies.

In conclusion, in this large study with comprehensive patient characterization, molecular characteristics of rectal tumors were not significantly associated with tumor response or pCR following neoadjuvant treatment. None of the patient characteristics investigated was predictive of response to nCRT. While we found no clear indication that KRAS mutations might be associated with response to nCRT, larger studies determining molecular markers before the administration of neoadjuvant treatment are needed to investigate this more accurately. Such studies should also integrate information on other characteristic genetic changes of rectal cancer, such as APC and TP53 mutations.

Acknowledgments The authors would like to thank the study participants, the interviewers who collected the data, and the medical documentarists who process and maintain the study database. The authors also thank the following hospitals and cooperating pathology institutes, and other institutions that recruited patients for this study: Chirurgische Universitätsklinik Heidelberg, Klinik am Gesundbrunnen Heilbronn, St Vincentiuskrankenhaus Speyer, St Josefskrankenhaus Heidelberg, Chirurgische Universitätsklinik Mannheim, Diakonissenkrankenhaus Speyer, Krankenhaus Salem Heidelberg, Kreiskrankenhaus Schwetzingen, St Marienkrankenhaus Ludwigshafen, Klinikum Ludwigshafen, Stadtklinik Frankenthal, Diakoniekrankenhaus Mannheim, Kreiskrankenhaus Sinsheim, Klinikum am Plattenwald Bad Friedrichshall, Kreiskrankenhaus Weinheim, Kreiskrankenhaus Eberbach, Kreiskrankenhaus Buchen, Kreiskrankenhaus Mosbach, Enddarmzentrum Mannheim, Kreiskrankenhaus Brackenheim, and Cancer Registry of Rhineland-Palatinate, Mainz.

Authors' contributions Study concept and design: EA, JCC, $\mathrm{HBr}, \mathrm{MH}$; acquisition of data: EA, LJ, HBl, MK, KT, WR, EH, JCC, HBr, MH; analysis and interpretation of data: EA, LJ, JK, EAm, HBl, MK, KT, WR, $\mathrm{EH}, \mathrm{JCC}, \mathrm{HBr}, \mathrm{MH}$; statistical analysis: EA, MH; drafting of the manuscript: EA, MH; obtained funding: HBl, EH, WR, MK, JCC, HBr, MH; administrative, technical, or material support: $\mathrm{EH}, \mathrm{JCC}, \mathrm{HBr}, \mathrm{MH}$; study supervision: JCC, $\mathrm{HBr}, \mathrm{MH}$; critical revision of the manuscript for important intellectual content: all authors; approval of the final version of the manuscript: all authors.

Funding Open Access funding enabled and organized by Projekt DEAL. This work was supported by the German Research Council (BR 1704/61, BR 1704/6-3, BR 1704/6-4, CH 117/1-1, HO 5117/2-1, HE 5998/2-1, KL 2354/3-1, RO 2270/8-1, and BR 1704/17-1); the Interdisciplinary Research Program of the National Center for Tumor Diseases (NCT), Germany; and the German Federal Ministry of Education and Research (01KH0404, 01ER0814, 01ER0815, 01ER1505A, and 01ER1505B).

\section{Compliance with ethical standards}

Conflict of interest The authors declare that they have no conflict of interest.

Ethics approval and consent to participate This study was approved by the ethical committees of the Medical Faculty of the University of Heidelberg and the Medical Chambers of Baden-Württemberg and Rhineland-Palatinate. Written informed consent was obtained from each participant.

Disclaimer The funding organizations had no role on the design and conduct of the study; collection, management, analysis, and interpretation of the data; preparation, review, or approval of the manuscript; and decision to submit the manuscript for publication.

Open Access This article is licensed under a Creative Commons Attribution 4.0 International License, which permits use, sharing, adaptation, distribution and reproduction in any medium or format, as long as you give appropriate credit to the original author(s) and the source, provide a link to the Creative Commons licence, and indicate if changes were made. The images or other third party material in this article are included in the article's Creative Commons licence, unless indicated 
otherwise in a credit line to the material. If material is not included in the article's Creative Commons licence and your intended use is not permitted by statutory regulation or exceeds the permitted use, you will need to obtain permission directly from the copyright holder. To view a copy of this licence, visit http://creativecommons.org/licenses/by/4.0/.

\section{References}

1. National Comprehensive Cancer Network. Rectal Cancer. Version 1.20192019 [updated March 15, 2019. Available from: https:// www.nccn.org/professionals/physician_gls/pdf/rectal.pdf.

2. Paun BC, Cassie S, MacLean AR, Dixon E, Buie WD (2010) Postoperative complications following surgery for rectal cancer. Ann Surg 251(5):807-818

3. Juul T, Ahlberg M, Biondo S, Espin E, Jimenez LM, Matzel KE, Palmer GJ, Sauermann A, Trenti L, Zhang W, Laurberg S, Christensen P (2014) Low anterior resection syndrome and quality of life: an international multicenter study. Dis Colon Rectum 57(5): 585-591

4. Hupkens BJP, Martens MH, Stoot JH, Berbee M, Melenhorst J, Beets-Tan RG, Beets GL, Breukink SO (2017) Quality of life in rectal cancer patients after chemoradiation: watch-and-wait policy versus standard resection-a matched-controlled study. Dis Colon Rectum 60(10):1032-1040

5. Habr-Gama A, Perez RO, Nadalin W et al (2004) Operative versus nonoperative treatment for stage 0 distal rectal cancer following chemoradiation therapy: long-term results. Ann Surg 240(4):711717 discussion 7-8

6. van der Valk MJM, Hilling DE, Bastiaannet E, Meershoek-Klein Kranenbarg E, Beets GL, Figueiredo NL, Habr-Gama A, Perez RO, Renehan AG, van de Velde CJH, Ahlberg M, Appelt A, Asoglu O, Bär MT, Barroca R, Beets-Tan RGH, Belgers EHJ, Bosker RJI, Breukink SO, Bujko K, Carvalho C, Cunningham C, Creavin B, D'Hoore A, Gérard JP, Gollins S, Hoff C, Holman FA, Hupkens BJP, Iseas S, Jakobsen A, Keshvari A, Koopal SA, Kusters M, Langheinrich M, Leijtens JWA, Maas M, Malcomson L, Mamedli ZZ, Martling A, Matzel KE, Melenhorst J, Morici ML, Murad-Regadas SM, O'Dwyer ST, Peeters KCMJ, Rosa I, Rossi G, Rutten HJT, Sanchez Loria F, van der Sande ME, São Julião GP, Saunders M, Sun Myint A, van der Sluis H, Schiappa R, Scott N, Stoot JHMB, Talsma AK, Terrasson I, Tokmak H, Vaccaro CA, Vahrmeijer AL, Wasowicz DK, Westreenen HL, Winter DC, Wolthuis AM, Zimmerman DDE (2018) Long-term outcomes of clinical complete responders after neoadjuvant treatment for rectal cancer in the International Watch \& Wait Database (IWWD): an international multicentre registry study. Lancet. 391(10139):25372545

7. Maas M, Beets-Tan RG, Lambregts DM et al (2011) Wait-and-see policy for clinical complete responders after chemoradiation for rectal cancer. J Clin Oncol 29(35):4633-4640

8. Martens MH, Maas M, Heijnen LA et al (2016) Long-term outcome of an organ preservation program after neoadjuvant treatment for rectal cancer. J Natl Cancer Inst 108(12):djw171

9. Renehan AG, Malcomson L, Emsley R, Gollins S, Maw A, Myint AS, Rooney PS, Susnerwala S, Blower A, Saunders MP, Wilson MS, Scott N, O'Dwyer ST (2016) Watch-and-wait approach versus surgical resection after chemoradiotherapy for patients with rectal cancer (the OnCoRe project): a propensity-score matched cohort analysis. Lancet Oncol 17(2):174-183

10. Dossa F, Chesney TR, Acuna SA, Baxter NN (2017) A watch-andwait approach for locally advanced rectal cancer after a clinical complete response following neoadjuvant chemoradiation: a systematic review and meta-analysis. Lancet Gastroenterol Hepatol 2(7):501-513

11. Chow OS, Kuk D, Keskin M, Smith JJ, Camacho N, Pelossof R, Chen CT, Chen Z, Avila K, Weiser MR, Berger MF, Patil S, Bergsland E, Garcia-Aguilar J (2016) KRAS and combined KRAS/TP53 mutations in locally advanced rectal cancer are independently associated with decreased response to neoadjuvant therapy. Ann Surg Oncol 23(8):2548-2555

12. Russo AL, Ryan DP, Borger DR, Wo JY, Szymonifka J, Liang WY, Kwak EL, Blaszkowsky LS, Clark JW, Allen JN, Zhu AX, Berger DL, Cusack JC, Mamon HJ, Haigis KM, Hong TS (2014) Mutational and clinical predictors of pathologic complete response in the treatment of locally advanced rectal cancer. J Gastrointest Cancer 45(1):34-39

13. Chow OS, Smith JJ, Gollub MJ, Garcia-Aguilar J (2014) Can we predict response and/or resistance to neoadjuvant chemoradiotherapy in patients with rectal cancer? Curr Color Cancer Rep 10(2): 164-172

14. Sottoriva A, Kang H, Ma Z, Graham TA, Salomon MP, Zhao J, Marjoram P, Siegmund K, Press MF, Shibata D, Curtis C (2015) A Big Bang model of human colorectal tumor growth. Nat Genet 47(3):209-216

15. Blaker H, Alwers E, Arnold A et al (2019) The association between mutations in BRAF and colorectal cancer-specific survival depends on microsatellite status and tumor stage. Clin Gastroenterol Hepatol $17: 455-462$

16. Brenner H, Jansen L, Ulrich A, Chang-Claude J, Hoffmeister M (2016) Survival of patients with symptom- and screening-detected colorectal cancer. Oncotarget. 7(28):44695-44704

17. Alwers E, Blaker H, Walter V et al (2019) External validation of molecular subtype classifications of colorectal cancer based on microsatellite instability, CIMP, BRAF and KRAS. BMC Cancer 19(1):681

18. Charlson ME, Pompei P, Ales KL, MacKenzie CR (1987) A new method of classifying prognostic comorbidity in longitudinal studies: development and validation. J Chronic Dis 40(5):373-383

19. Quan H, Sundararajan V, Halfon P, Fong A, Burnand B, Luthi JC, Saunders LD, Beck CA, Feasby TE, Ghali WA (2005) Coding algorithms for defining comorbidities in ICD-9-CM and ICD-10 administrative data. Med Care 43(11):1130-1139

20. R Core Team. R: a language and environment for statistical computing. Vienna, Austria: R Foundation for Statistical Computing; 2018.

21. Das P, Skibber JM, Rodriguez-Bigas MA, Feig BW, Chang GJ, Wolff RA, Eng C, Krishnan S, Janjan NA, Crane CH (2007) Predictors of tumor response and downstaging in patients who receive preoperative chemoradiation for rectal cancer. Cancer. 109(9):1750-1755

22. Collette L, Bosset JF, den Dulk M, Nguyen F, Mineur L, Maingon P, Radosevic-Jelic L, Piérart M, Calais G, European Organisation for Research and Treatment of Cancer Radiation Oncology Group (2007) Patients with curative resection of cT3-4 rectal cancer after preoperative radiotherapy or radiochemotherapy: does anybody benefit from adjuvant fluorouracil-based chemotherapy? A trial of the European Organisation for Research and Treatment of Cancer Radiation Oncology Group. J Clin Oncol 25(28):4379-4386

23. Park IJ, You YN, Agarwal A, Skibber JM, Rodriguez-Bigas MA, Eng C, Feig BW, Das P, Krishnan S, Crane CH, Hu CY, Chang GJ (2012) Neoadjuvant treatment response as an early response indicator for patients with rectal cancer. J Clin Oncol 30(15):1770 1776

24. Maas M, Nelemans PJ, Valentini V, Das P, Rödel C, Kuo LJ, Calvo FA, García-Aguilar J, Glynne-Jones R, Haustermans K, Mohiuddin M, Pucciarelli S, Small W Jr, Suárez J, Theodoropoulos G, Biondo S, Beets-Tan RGH, Beets GL (2010) Long-term outcome in patients with a pathological complete response after chemoradiation 
for rectal cancer: a pooled analysis of individual patient data. Lancet Oncol 11(9):835-844

25. Lichthardt S, Zenorini L, Wagner J, Baur J, Kerscher A, Matthes N, Kastner C, Pelz J, Kunzmann V, Germer CT, Wiegering A (2017) Impact of adjuvant chemotherapy after neoadjuvant radio- or radiochemotherapy for patients with locally advanced rectal cancer. J Cancer Res Clin Oncol 143(11):2363-2373

26. Lichthardt S, Wagner J, Lob S et al (2020) Pathological complete response due to a prolonged time interval between preoperative chemoradiation and surgery in locally advanced rectal cancer: analysis from the German StuDoQ $\mid$ Rectalcarcinoma registry. BMC Cancer 20(1):49

27. Akce M, El-Rayes BF (2019) Nonsurgical management of rectal cancer. J Oncol Pract 15(3):123-131

28. Dayde D, Tanaka I, Jain R, Tai MC, Taguchi A (2017) Predictive and prognostic molecular biomarkers for response to neoadjuvant chemoradiation in rectal cancer. Int J Mol Sci 18(3):573

29. Gaedcke J, Grade M, Jung K, Schirmer M, Jo P, Obermeyer C, Wolff HA, Herrmann MK, Beissbarth T, Becker H, Ried T, Ghadimi M (2010) KRAS and BRAF mutations in patients with rectal cancer treated with preoperative chemoradiotherapy. Radiother Oncol 94(1):76-81

30. Davies JM, Trembath D, Deal AM et al (2011) Phospho-ERK and AKT status, but no KRAS mutation status, are associated with outcomes in rectal cacner treated with chemoradiotherapy. Radiother Oncol 6:114

31. Duldulao MP, Lee W, Nelson RA, Li W, Chen Z, Kim J, GarciaAguilar J (2013) Mutations in specific codons of the KRAS oncogene are associated with variable resistance to neoadjuvant chemoradiation therapy in patients with rectal adenocarcinoma. Ann Surg Oncol 20(7):2166-2171

32. Frydrych LM, Ulintz P, Bankhead A, Sifuentes C, Greenson J, Maguire L, Irwin R, Fearon ER, Hardiman KM (2019) Rectal cancer sub-clones respond differentially to neoadjuvant therapy. Neoplasia. 21(10):1051-1062
33. Janjan NA, Crane C, Feig BW, Cleary K, Dubrow R, Curley S, Vauthey JN, Lynch P, Ellis LM, Wolff R, Lenzi R, Abbruzzese J, Pazdur R, Hoff PM, Allen P, Brown T, Skibber J (2001) Improved overall survival among responders to preoperative chemoradiation for locally advanced rectal cancer. Am J Clin Oncol 24(2):107-112

34. Fokas E, Liersch T, Fietkau R, Hohenberger W, Beissbarth T, Hess C, Becker H, Ghadimi M, Mrak K, Merkel S, Raab HR, Sauer R, Wittekind C, Rödel C (2014) Tumor regression grading after preoperative chemoradiotherapy for locally advanced rectal carcinoma revisited: updated results of the CAO/ARO/AIO-94 trial. J Clin Oncol 32(15):1554-1562

35. Fokas E, Strobel P, Fietkau R et al (2017) Tumor regression grading after preoperative chemoradiotherapy as a prognostic factor and individual-level surrogate for disease-free survival in rectal cancer. J Natl Cancer Inst 109(12). https://doi.org/10.1093/jnci/djx095

36. Bernier L, Balyasnikova S, Tait D, Brown G (2018) Watch-andwait as a therapeutic strategy in rectal cancer. Curr Colorectal Cancer Rep 14(2):37-55

37. On J, Aly EH (2018) 'Watch and wait' in rectal cancer: summary of the current evidence. Int J Colorectal Dis 33(9):1159-1168

38. Hiotis SP, Weber SM, Cohen AM, Minsky BD, Paty PB, Guillem JG, Wagman R, Saltz LB, Wong WD (2002) Assessing the predictive value of clinical complete response to neoadjuvant therapy for rectal cancer: an analysis of 488 patients. J Am Coll Surg 194(2): $131-136$

39. Glynne-Jones R, Wallace M, Livingstone JI, Meyrick-Thomas J (2008) Complete clinical response after preoperative chemoradiation in rectal cancer: is a "wait and see" policy justified? Dis Colon Rectum 51(1):10-19 discussion 9-20

Publisher's note Springer Nature remains neutral with regard to jurisdictional claims in published maps and institutional affiliations.

\section{Affiliations}

\section{Elizabeth Alwers ${ }^{1}$. Lina Jansen ${ }^{1}$ • Jakob Kather ${ }^{2}$ - Efrat Amitay ${ }^{1} \cdot$ Hendrik Bläker $^{3}$ • Matthias Kloor ${ }^{4}$. Katrin E. Tagscherer ${ }^{5,6}$. Wilfried Roth ${ }^{5,6} \cdot$ Esther Herpel $^{5,7}$ • Jenny Chang-Claude ${ }^{8,9} \cdot$ Hermann Brenner $^{1,10,11}$. Michael Hoffmeister ${ }^{1}$}

1 Division of Clinical Epidemiology and Aging Research, German Cancer Research Center (DKFZ), Im Neuenheimer Feld 581, 69120 Heidelberg, Germany

2 Department of Medicine III, University Hospital RWTH Aachen, Aachen, Germany

3 Institute of Pathology, University Hospital Leipzig, Leipzig, Germany

4 Department of Applied Tumor Biology, Institute of Pathology, University of Heidelberg, Heidelberg, Germany

5 Institute of Pathology, University Hospital Heidelberg, Heidelberg, Germany

6 Institute of Pathology, University Medical Center Mainz, Mainz, Germany
7 NCT Tissue Bank, National Center for Tumor Diseases (NCT), Heidelberg, Germany

8 Division of Cancer Epidemiology, German Cancer Research Center (DKFZ), Heidelberg, Germany

9 Genetic Tumor Epidemiology Group, University Medical Center Hamburg-Eppendorf, Hamburg, Germany

10 Division of Preventive Oncology, German Cancer Research Center (DKFZ), National Center for Tumor Diseases (NCT), Heidelberg, Germany

11 German Cancer Consortium (DKTK), German Cancer Research Center (DKFZ), Heidelberg, Germany 